\title{
ESTUDO HIDROQUÍMICO E ISOTÓPICO DAS ÁGUAS SUBTERRÂNEAS NA PORÇÃO CENTRAL DO AQUÍFERO TUBARÃO, ESTADO DE SÃO PAULO - RESULTADOS PRELIMINARES
}

\author{
Sibele Ezaki ${ }^{1}$; Mara Akie Iritani ${ }^{1}$; Geraldo Hideo Oda ${ }^{1}$; Didier Gastmans ${ }^{2}$; Sueli Yoshinaga- \\ Pereira $^{3}$
}

\section{RESUMO}

Este trabalho apresenta resultados parciais de estudo hidroquímico e isotópico do Aquífero Tubarão, no Estado de São Paulo, e tem como proposta compreender a circulação das águas subterrâneas a partir da aplicação de isótopos ambientais (18O, 2H, 14C). Idades estimadas de radiocarbono de 15 amostras deste aquífero foram determinadas e variaram entre 1.897 anos A.P. (em Capivari) e 44.763 A.P. (em Monte Mor). Com a classificação hidroquímica das águas de 49 poços, foram reconhecidos 3 grupos: bicarbonatadas cálcicas, bicarbonatadas sódicas-cálcicas (mistas) e bicarbonatadas sódicas. Os dois primeiros grupos apresentam características próximas, com pH neutro a alcalino, composição isotópica média mais enriquecida $\left(\delta^{2} \mathrm{H}=-47,68 \%\right.$ e $\delta^{18} \mathrm{O}=-7,44 \%$ ) e idades mais jovens que 10.000 A.P. O terceiro grupo está associado a águas alcalinas, maior profundidade média de poço (325 m), composição isotópica mais empobrecida $\left(\delta^{2} \mathrm{H}=-58,37 \%\right.$ e $\delta^{18} \mathrm{O}=-8,65 \%$ o $)$ e abrange amostras de idades mais antigas que 10.000 A.P. A elevada profundidade do poço nem sempre implica em maior idade da água, podendo esta ser mais influenciada pela geometria das camadas e litologias heterogêneas que interferem na circulação do fluxo subterrâneo.

\section{ABSTRACT}

This paper presents results of an hydrochemical and isotopic study of the Tubarão Aquifer, in the state of São Paulo, and aims to understand the groundwater circulations applying environmental isotopes (18O, 2H, 14C). Estimated radiocarbon ages of 15 samples of this aquifer were determined and ranged from 1,897 B.P. years (in Capivari) and 44,763 B.P. (in Monte Mor). By the use of hydrochemical classification of 49 wells water, three groups were recognized: calcium bicarbonate, sodium-calcium bicarbonate (mixed) and sodium bicarbonate. The first two groups have similar characteristics, with neutral to alkaline $\mathrm{pH}$, more enriched average isotopic composition $\left(\delta^{2} \mathrm{H}=\right.$ -

\footnotetext{
${ }^{1}$ Instituto Geológico, Secretaria do Meio Ambiente do Estado de São Paulo. R. Joaquim Távora, 822, Vila Mariana, São Paulo-SP, CEP 04015-011. Fone: (11) 5073-5511. E-mails: sibezaki@gmail.com, mara.iritani@terra.com.br, ghoda1947@gmail.com

${ }^{2}$ Cento de Estudos Ambientais, Universidade Estadual PaulistaJúlio de Mesquita Filho-UNESP, Campus Rio Claro. Av. 24-A, 1515, Bairro Bela Vista, Rio Claro-SP, CEP: 13506-900. Fone: (19) 3526-9496. E-mail: gastmans@rc.unesp.br

3 Instituto de Geociências, Universidade Estadual de Campinas - UNICAMP. R. João Pandiá Calógeras, 51, Campinas-SP, CEP: 13083-870. Fone: (19) 3521-4698. E-mail: sueliyos@ige.unicamp.br
} 
$47,68 \%$ o $\delta^{18} \mathrm{O}=-7,44 \%$ ) and ages younger than 10,000 B.P. The third group is associated to alkaline waters, higher average well depth (325 m), more negative isotopic composition $\left(\delta^{2} \mathrm{H}=-58,37 \%\right.$ e $\delta^{18} \mathrm{O}=-8,65 \%$ o) and includes samples of older than 10,000 B.P. ages. The higher well depth does not necessarily imply a greater water age, which may be more influenced by the geometry of layers and heterogeneous lithology that interfere in the circulation of groundwater flow.

Palavras-chave: Aquífero Tubarão, isótopos estáveis, idade radiocarbono 


\section{INTRODUÇÃO}

O Aquífero Tubarão (AT) no Estado de São Paulo é uma unidade hidrogeológica basal da Bacia do Paraná, de baixa produtividade, com vazões predominantemente inferiores a $10 \mathrm{~m}^{3} / \mathrm{h}$ (DAEE/IG/IPT/CPRM, 2005), associada à heterogeneidade litológica e descontinuidade das camadas geológicas, tanto lateral como verticalmente (DAEE,1981, 1982). Constitui, entretanto, significativa fonte de abastecimento público e privado, em função da crescente demanda por água subterrânea em importantes centros urbanos, como por exemplo, na Região Metropolitana de Campinas (Oda et al. 2013).

Estudos hidrogeológicos averiguaram a ocorrência de porções do aquífero mais produtivas (Q> $40 \mathrm{~m}^{3} / \mathrm{h}$ ) em alguns municípios (como Tietê, Capivari, Elias Fausto, Iperó, Araras-Casa Branca, entre outros), mas também indícios de rebaixamentos da superfície potenciométrica devido ao bombeamento intensivo dos poços (Capivari, Rafard, Tietê, Hortolândia) (DAEE/IG/IPT/CPRM, 2005; Ferreira et al., 2005; Iritani et al., 2009; Oda et al., 2013). Esses estudos apontam para a necessidade de uma melhor avaliação do potencial produtivo do AT frente às pressões, e aprofundar o conhecimento sobre a circulação das águas subterrâneas e mecanismos de recarga, visando sua sustentabilidade. Como a heterogeneidade litológica do aquífero deve refletir na recarga, a exploração intensiva das porções mais profundas (superiores a $200 \mathrm{~m}$ ), por vezes sotopostas e interdigitadas a camadas de sedimentos menos permeáveis, pode levar ao esgotamento das camadas mais produtivas.

Este trabalho tem como proposta aprimorar o conhecimento hidrogeológico da porção central do AT no Estado de São Paulo, a partir de estudos geoquímicos e isotópicos, com o objetivo de investigar os mecanismos que controlam a composição química e a circulação das águas profundas do AT. O estudo do potencial de recarga do AT, a partir da aplicação de técnicas de datação utilizando o 14C, objetiva verificar a ocorrência de águas antigas, que podem representar recursos nãorenováveis, visando estabelecer áreas críticas (mais sensíveis) que necessitem de medidas de proteção evitando a exploração indiscriminada e esgotamento dos recursos. Como objetivos específicos este estudo pretende: 1) estabelecer uma linha de base da composição isotópica a partir da qual se acompanhe a evolução da composição e idades das águas subterrâneas ao longo das décadas; 2) utilizar a cronologia isotópica para melhoria da gestão dos recursos hídricos.

\section{CARACTERIZAÇÃO DA ÁREA INVESTIGADA}

A área de estudo abrange 15 municípios que estão situados na Unidade de Gerenciamento de Recursos Hídricos das Bacias dos rios Piracicaba, Capivari e Jundiaí (UGRHI 5-PCJ) e em parte do Sorocaba/Médio Tietê (UGRHI 10) (Figura 1). O limite da área é o mesmo considerados no estudo 
hidrogeológico de Vidal (2002) (UTM 7482kmN/300kmE; 7420kmN/218kmE zona 23S), onde também estão abrangidos outros estudos como os de Maniakas (1986), Stevaux et al.(1987), Diniz (1990), IG (1990, 1991, 1993, 1995), Yoshinaga-Pereira (1997), Silva (2000), Vidal et al. (2005), Ezaki (2011), Oda et al. (2013), Montanheiro (2014).

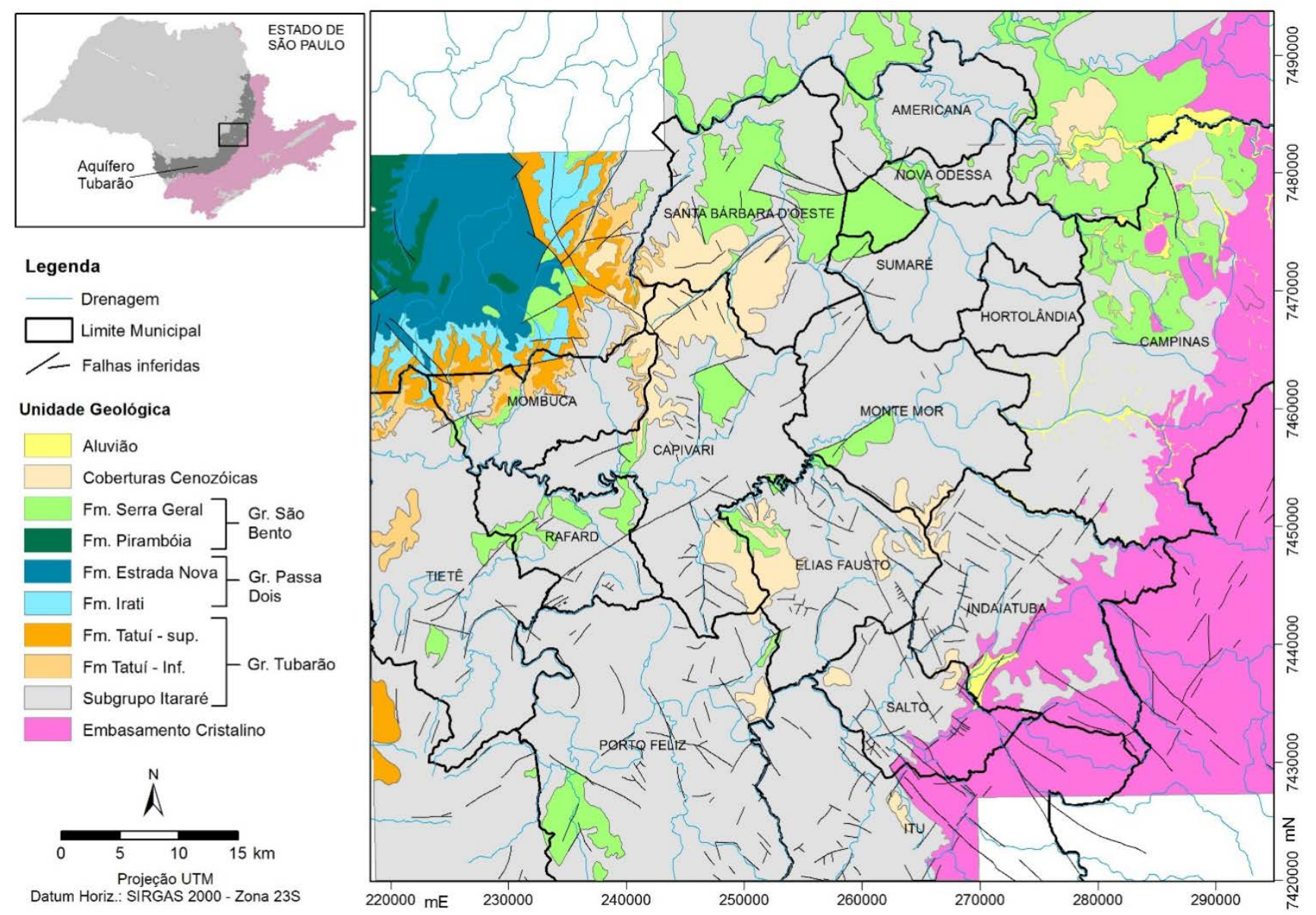

Figura 1. Mapa geológico da área de estudo (Fontes: DAEE/UNESP, 1980; Teixeira et al., 2009).

No contexto geológico, a área de estudo localiza-se na porção leste da Bacia Sedimentar do Paraná. O Grupo Tubarão, idade permocabonífera e unidade de interesse no estudo, sobrepõe-se ao Embasamento Cristalino pré-Cambriano, e apresenta intrusões de corpos isolados de diabásio. O Grupo Tubarão subdivide-se em duas unidades principais: Subgrupo Itararé e Formação Tatuí. Sua espessura chega a 1.300 m na região de Paraguaçu Paulista (SP) próximo ao centro da Bacia do Paraná; nas proximidades de Itu atinge 300 m, diminuindo expressivamente em direção a leste, apresentando-se em forma de cunha (Oda, 1998).

Foi depositado em ambientes lacustres, deltaicos e marinhos presentes na interface entre o continente e a plataforma marinha, sob influência glacial, periglacial e interglacial (Zalán et al., 1991; Petri e Pires, 1992; Santos, 1996, Salvetti, 2005). Constitui-se de litotipos bastante variáveis e 
descontínuos representados por intercalações e associações de arenitos, siltitos, folhelhos, argilitos, diamictitos e ritmitos, com variação e interdigitação faciológica, tanto horizontal como verticalmente.

O Aquífero Tubarão constitui um aquífero livre com características locais de semiconfinamento devido à disposição irregular de camadas e lentes de sedimentos finos (lamito, siltito e folhelho) em meio a sedimentos clásticos grossos (arenito, conglomerado e diamictito), conforme ambiente de deposição. Isolamento de corpos arenosos, baixa permeabilidade devido a presença de lentes de argila e baixa porosidade dificultam o fluxo vertical da água, conferindo-lhe características anisotrópicas (DAEE, 1981, 1982).

O fluxo regional das águas subterrâneas é de Epara W, da borda para o interior da Bacia do Paraná, podendo apresentar direção N-S próximo a vales dos principais rios da região (Vidal, 2002), como os rios Capivari e Tietê, e rio Sorocaba a sul da área estudada (Oda et al., 2013).

\section{MÉTODOS}

Para a execução do estudo foram realizadas as seguintes atividades:

- Levantamento de dados e informações de poços. Os dados de poços que atravessam o Aquífero Tubarão foram levantados: a) no cadastro de poços do Instituto Geológico da Secretaria do Meio Ambiente do Estado de São Paulo; b) em projetos de pesquisa, como do DAEE-UNESP (2013); c) no cadastro físico de poços outorgados pelo Departamento de Águas e Energia Elétrica do Estado de São Paulo (DAEE). Os dados cadastrais de poços foram avaliados quanto à qualidade da informação (litologia, perfil construtivo, teste de vazão, localização, etc.) e compuseram um banco de dados. Serviram de base para a análise hidrogeológica e para a seleção dos poços a serem amostrados.

- Tratamento de dados hidrogeológicos. O mapa potenciométrico foi elaborado a partir de dados de altitude do nível estático (NE), obtidos nos relatórios de poços cadastrados. Os dados foram interpolados utilizando o método de krigagem, pelo programa Surfer (Goldensoftware), v.12.0.

- Seleção dos poços. As amostras foram coletadas preferencialmente em poços de concessionárias de abastecimento público. Nos municípios onde não há abastecimento público, foram amostrados poços em indústrias, que normalmente apresentam relatórios de construção de poços com dados confiáveis de litologia, testes de vazão e construtivos.

- Amostragens. Foram efetuadas no período de março/2015 a maio/2016 em 49 poços conforme normas estabelecidas pelo Standard Methods for the Examination of Water and Wastewater (APHA/AWWA/WEF, 1995). As amostras de água subterrânea foram coletadas na saída do poço, após período de 5 a 10 minutos de bombeamento e acondicionadas em frascos plásticos de polietileno de alta densidade (PEAD). Logo após a coleta, foram analisados in situ os parâmetros pH, Eh, condutividade elétrica e temperatura. As amostras foram filtradas (acetato 0,45 $\mu \mathrm{m}$ ), armazenadas em 
frascos de PEAD e mantidas à temperatura inferior a $4^{\circ} \mathrm{C}$ para encaminhamento aos laboratórios de análises hidroquímicas. A preservação de amostras para análise de cátions foi efetuada com $\mathrm{HNO}_{3}$ até a solução atingir $\mathrm{pH}<2$. As alcalinidades total e parcial foram determinadas por titulometria. Alíquotas para análise isotópica de ${ }^{18} \mathrm{O},{ }^{2} \mathrm{H}$ e radiocarbono foram coletadas e armazenadas em frasco de vidro âmbare acondicionadas à temperatura ambiente.

- Análises laboratoriais. As amostras foram encaminhadas ao Laboratório de Hidrogeologia e Hidroquímica, Instituto de Geociências e Ciências Exatas (UNESP, Rio Claro), para determinação de cátions por espectrometria de emissão atômica com fonte de plasma de argônio indutivo ICPAES)(Al, Ba, Ca, Fe total, Mg, Mn, Si)e cromatografia iônica $\left(\mathrm{Na}^{+}, \mathrm{K}^{+}\right)$e de ânions por cromatografia iônica $\left(\mathrm{F}^{-}, \mathrm{Cl}^{-}, \mathrm{NO}_{3}{ }^{-}, \mathrm{SO}_{4}{ }^{2-}\right)$. As análises isotópicas $(\delta 2 \mathrm{H}$ e $\delta 18 \mathrm{O})$ foram realizadas no Laboratório de Isótopos Estáveis do Departamento de Geologia Aplicada (UNESP, Rio Claro), pela técnica de espectroscopia a laser empregando um analisador de isótopos, modelo LWIA-24-EP da Los Gatos Research. Do total de amostras, 15 foram encaminhadas para análise da razão $\delta 13 \mathrm{C} / 14 \mathrm{C}$ e datação por radiocarbono via espectrometria de massa com aceleradores (AMS) no Environmental Isotope Laboratory - Earth \& Environmental Sciences Department, da Universidade de Waterloo.

\section{RESULTADOS E DISCUSSÕES}

O levantamento de dados de poços resultou em um banco de dados com 1678 registros, dentre os quais 1312 explotam somente o Aquífero Tubarão. A profundidade média destes é de 200 m na região estudada.

As profundidades média e mediana dos 49 poços amostrados são, respectivamente, 277 m e 286m, com valor mínimo de 118 m e máximo de $450 \mathrm{~m}$. Os poços mais profundos encontram-se em Porto Feliz (450 m), Sumaré (430 m),Americana (447) e Capivari (400 m), alguns atingindo o Embasamento Cristalino.

Na Figura 2 é apresentado o mapa potenciométrico e a localização dos 49 poços amostrados. Há uma tendência regional do fluxo subterrâneo de E para W, mas localmente o sentido pode ser alterado para as áreas de descargas que correspondem aos principais rios da região, como o Ribeirão dos Quilombos (Campinas, Hortolândia, Sumaré, Nova Odessa), Rio Capivari (Capivari, Rafard), e Rio Tietê (Salto, Tietê).Entre os municípios de Campinas e Americana, a norte da região estudada, o fluxo apresenta sentido de SE para NW em direção ao rio Piracicaba. 


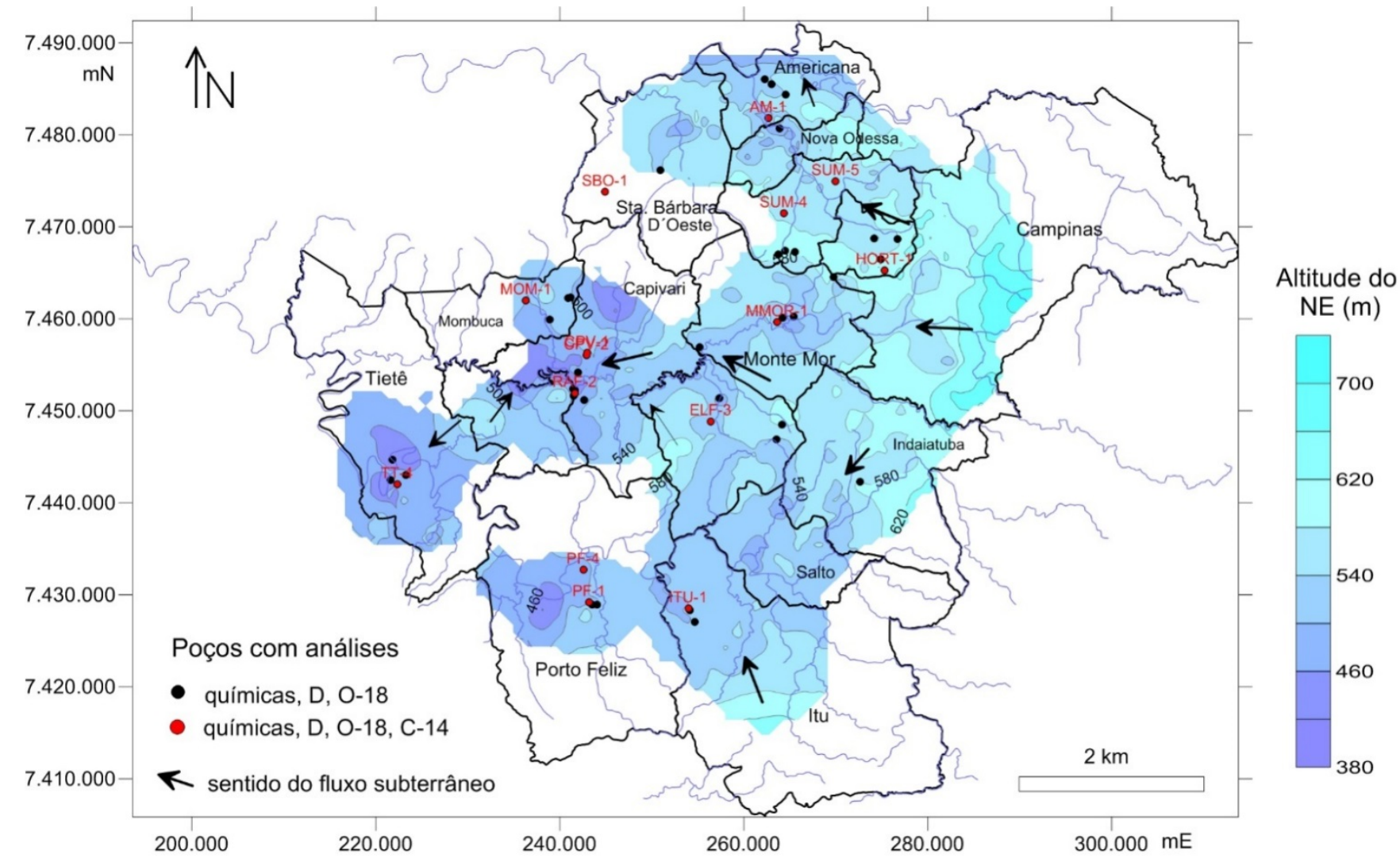

Figura 2. Mapa potenciométricoda área de estudo e localização dos poços amostrados.

\section{Hidroquímica}

O pH médio das amostras é 8,12, alcalino, variando de 6,82 a 9,26. Os valores de pH das amostras IND-1 ( $\mathrm{pH}=4,72)$ e HORT-4 $(\mathrm{pH}=5,55)$ foram desconsiderados, uma vez que apresentam indicação de infiltração de efluentes dadas as concentrações de nitrato e cloreto, além de concentrações de $\mathrm{HCO}_{3}{ }^{-}$e condutividade elétrica características de água de chuva ou de água tratada.

A condutividade elétrica média das águas é de $269 \mu \mathrm{S} \mathrm{cm}^{-1}$, variando de 114 a $458 \mu \mathrm{S} \mathrm{cm}^{-1}$.

Pela classificação de Piper (Figura 3) há três grupos hidroquímicos em igual proporção considerando 47amostras:

1) águas bicarbonatadas cálcicas a cálcicas-sódicas (Ca-Mg-HCO 3 , Ca-Na-HCO, $\mathrm{Ca}-\mathrm{Na}-\mathrm{Mg}-$ $\mathrm{HCO}_{3}$ ) - 17 amostras: AM-2, AM-3, CPV-2, ELF-1, HORT-3, ITU-1, ITU-2, ITU-3, MOM-3, PF-3, PF-4, SOB-1, SOB-2, SUM-3, SUM-5, TT-2, TT-4 (círculos azuis);

2)águas bicarbonatadas sódicas-cálcicas (Na-Ca-HCO 3 )- 14 amostras: AM-4, CPV-1, ELF-2, ELF-3, HORT-1, MMOR-5B, MOM-2, MOM-4, NO-1, SUM-1, SUM-2, SUM-4, TT-1, TT-3 (círculos vermelhos);

3) águas bicarbonatadas sódicas (Na-HCO 3 ) 16 amostras: AM-1, CPV-3, CPV-4, HORT-2, MMOR-1, MMOR-3, MMOR-4, MOM-1, PF-1, PF-2, RAF-1, RAF-2, RAF-3, RAF-4 (círculos verdes). 


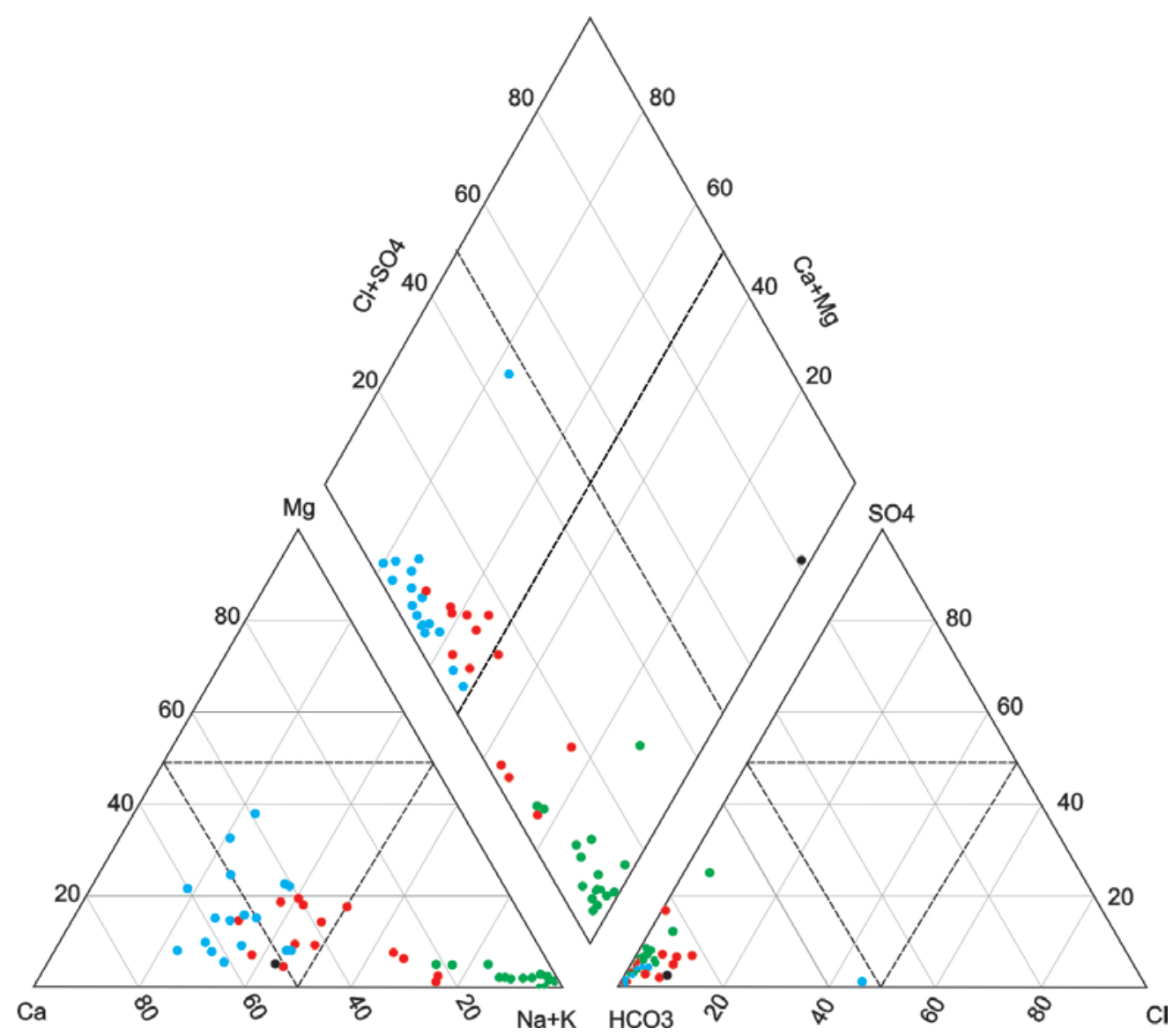

Figura 3. Diagrama de classificação hidroquímica - Piper das águas subterrâneas.

Há um aumento da profundidade média dos poços quando se consideram estes três grupos: 259,7m; 263,2 m e 325,5m, respectivamente.

O primeiro grupo se destaca nas concentrações médias mais elevadas de $\mathrm{Ca}^{2+}\left(33,5 \mathrm{mg} \mathrm{dm}^{-3}\right)$, $\mathrm{Mg}^{2+}\left(6,06 \mathrm{mg} \mathrm{dm}^{-3}\right), \mathrm{HCO}_{3}{ }^{-}\left(179,38 \mathrm{mg} \mathrm{dm}^{-3}\right)$ e Si $\left(20,95 \mathrm{mg} \mathrm{dm}^{-3}\right)$. O pH médio é de 7,72

O segundo grupo apresenta características mais próximas às do grupo 1 , como $\mathrm{pH}$ médio de 7,70 e teores iônicos médios, sendo alguns deles intermediários entre os grupos 1 e 3, como os de $\mathrm{Ca}^{2+}\left(19,3 \mathrm{mg} \mathrm{dm}^{-3}\right), \mathrm{Mg}^{2+}\left(2,79 \mathrm{mg} \mathrm{dm}^{-3}\right), \mathrm{Na}^{+}\left(28,36 \mathrm{mg} \mathrm{dm}^{-3}\right)$, Si $\left(16,94 \mathrm{mg} \mathrm{dm}^{-3}\right)$ e $\mathrm{F}^{-}\left(0,42 \mathrm{mg} \mathrm{dm}^{-}\right.$ $\left.{ }^{3}\right)$. Algumas concentrações médias foram as mais elevadas, como $\mathrm{Cl}^{-}\left(2,50 \mathrm{mg} \mathrm{dm}{ }^{-3}\right), \mathrm{NO}_{3}^{-}(2,80 \mathrm{mg}$ $\left.\mathrm{dm}^{-3}\right), \mathrm{K}^{+}\left(1,29 \mathrm{mg} \mathrm{dm}^{-3}\right)$ e $\mathrm{Fe}_{(\mathrm{t})}\left(0,99 \mathrm{mg} \mathrm{dm}^{-3}\right)$.

Já no terceiro grupo (Na-HCO3), observa-se que a média de pH se eleva a 8,91, assim como há concentrações médias mais elevadas de $\mathrm{SO}_{4}{ }^{2-}\left(10,79 \mathrm{mg} \mathrm{dm}^{-3}\right), \mathrm{F}^{-}\left(0,42 \mathrm{mg} \mathrm{dm}^{-3}\right)$ e $\mathrm{Na}^{+}\left(69,68 \mathrm{mg} \mathrm{dm}^{-}\right.$ $\left.{ }^{3}\right)$, além de $\mathrm{CO}_{3}{ }^{2-}\left(11,09 \mathrm{mg} \mathrm{dm}^{-3}\right)$.

Ao se observar o diagrama de Piper (Figura 3), observa-se que algumas amostras do grupo 2 (MOM-4, EF-2, MOM-2 e MMOR-5B) poderiam ser reunidas com as amostras do grupo 3 (Na-HCO3), e que as demais amostras têm características mais próximas às do grupo 1 (Ca-Na-HCO3). 


\section{Istópos Estáveis e Idade Radiocaborno}

As concentrações de isótopos nas amostras de água variam de -63,50 \% a -39,70\%o para deutério (D) e de $-9,41 \%$ a $-6,25 \%$ para ${ }^{18} \mathrm{O}$.

Constatam-se pelo menos dois grupos distintos de amostras: um em que as razões isotópicas são mais negativas (médias de $\delta^{2} \mathrm{H}=-58,37 \%$ e $\delta^{18} \mathrm{O}=-8,65 \%$ ) e, outro, com razões mais enriquecidas (médias de $\delta^{2} \mathrm{H}=-47,68 \%$ e $\delta^{18} \mathrm{O}=-7,44 \%$ ). $\mathrm{O}$ primeiro apresenta correspondência com as amostras de águas bicabornatadas sódicas (grupo hidroquímico 3), e o segundo com os tipos bicarbonatados cálcicos e cálcico-sódicos (grupos 1 e 2, respectivamente) (Figura 4). No diagrama de correlação das composições isotópicas (Figura 4), observa-se claramente este agrupamento e um ordenamento em sequência das amostras dos grupos hidroquímicos 1, 2 e 3.

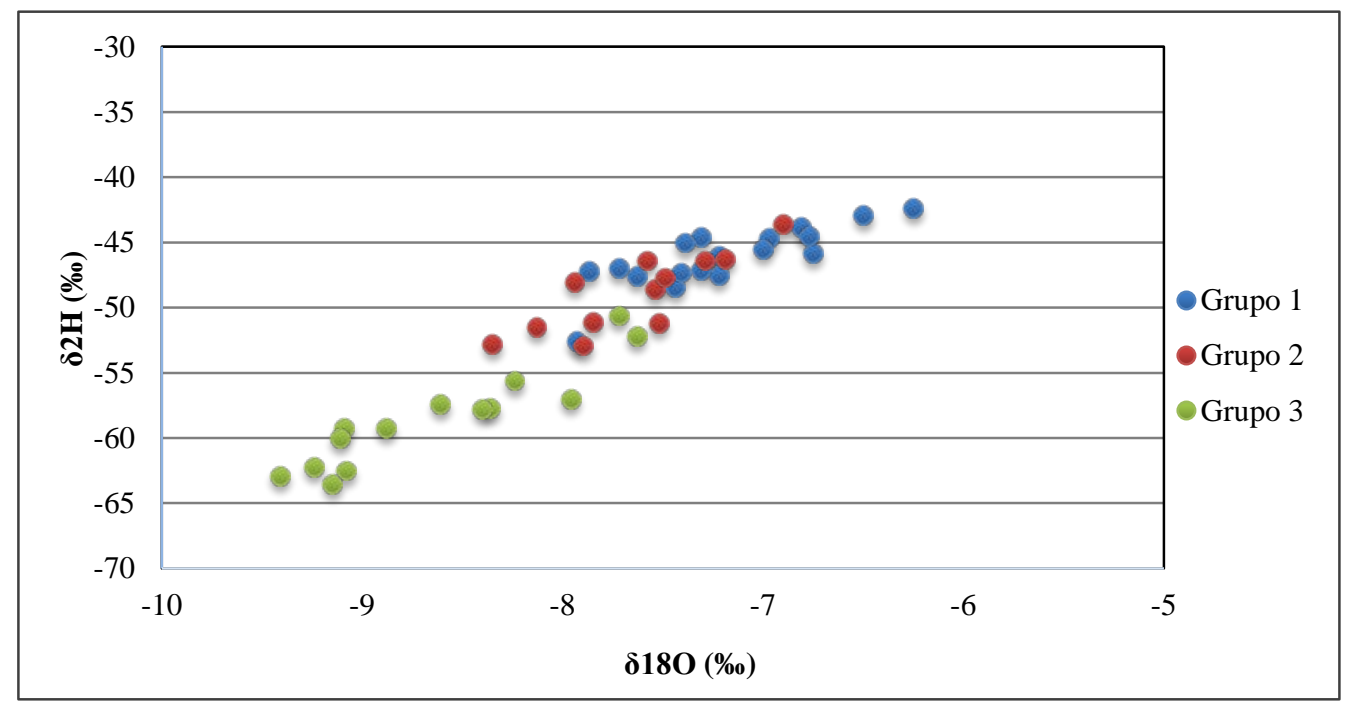

Figura 4. Correlação entre as composições dos isótopos estáveis ( $\left.\mathrm{D} X{ }^{18} \mathrm{O}\right)$ nas águas subterrâneas do AT e variação com relação aos tipos hidroquímicos.

Este agrupamento pode indicar variações climáticas durante suas épocas de recarga. Os dados mais empobrecidos poderiam estar relacionados a recarga sob condições climáticas mais frias, como também foi discutido por Aravena et al. (2005) e Hirata et al. (2011), em estudos de aquíferos confinados.

Na Figura 5 são correlacionados os dados de ${ }^{2} \mathrm{H}$ e ${ }^{18} \mathrm{O}$ das amostras de águas subterrâneas do AT, que se distribuem ao longo da reta meteórica mundial (tracejada). Nesta figura também é apresentada a distribuição de algumas amostras com relação às faixas de idades:

1) 0 a 5.000 anos A.P (em azul) - amostras SUM-4, SUM-5, HORT-1, CPV-2;

2) 5.000 a 10.000 anos A.P. (em vermelho) - amostras SOB-1, CPV-1, PF-4, TT-4, ELF-3;

3) 10.000 a 45.000 anos A.P. (em verde) - amostras AM-1, PF-1, SUM-3, MMOR-1. 
Constata-se uma correlação entre as idades estimadas com os valores isotópicos de ${ }^{2} \mathrm{H} \mathrm{e}{ }^{18} \mathrm{O}$. As amostras com idades mais antigas apresentam composições isotópicas mais empobrecidas.

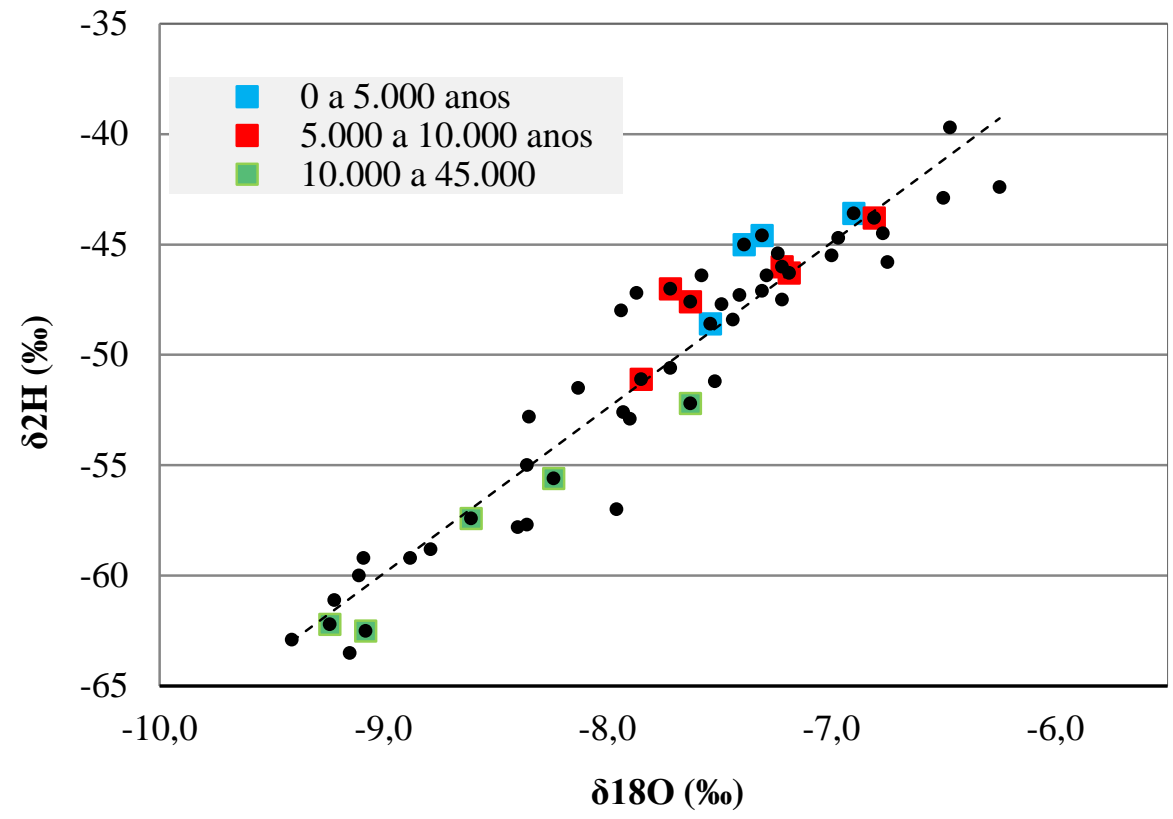

Figura 5. Correlação entre as composições isotópicas estáveis (O-18 X D) nas águas subterrâneas do AT e distribuição com relação às idades estimadas com C-14.

Na Figura 6 as correlações positivas entre composição isotópica de ${ }^{18} \mathrm{O}$ e teores do íon sódio $(\mathrm{R}=0,75)$, e entre teores de $\mathrm{Na}^{+}$e o $\mathrm{pH}(\mathrm{R}=0,77)$, confirmam a tendência das águas mais antigas estarem associadas a tipos mais sódicos (diagrama A), com pH alcalino (diagrama B).

A

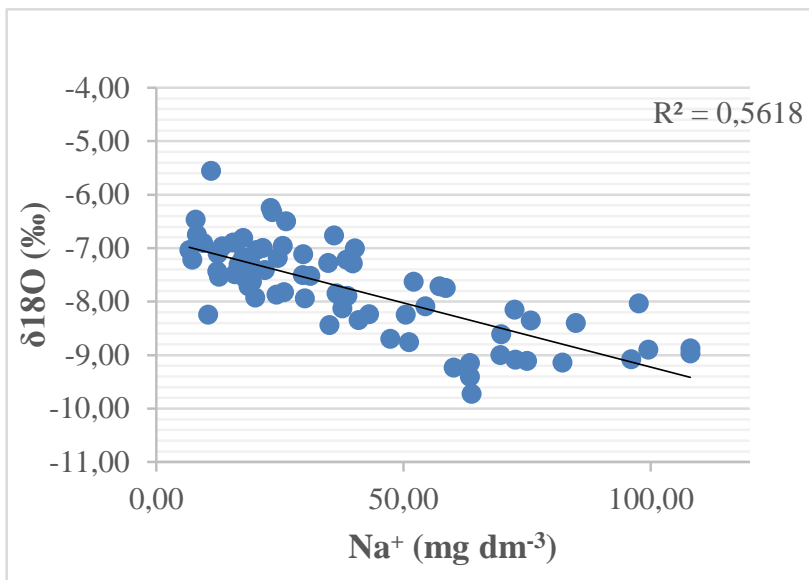

B

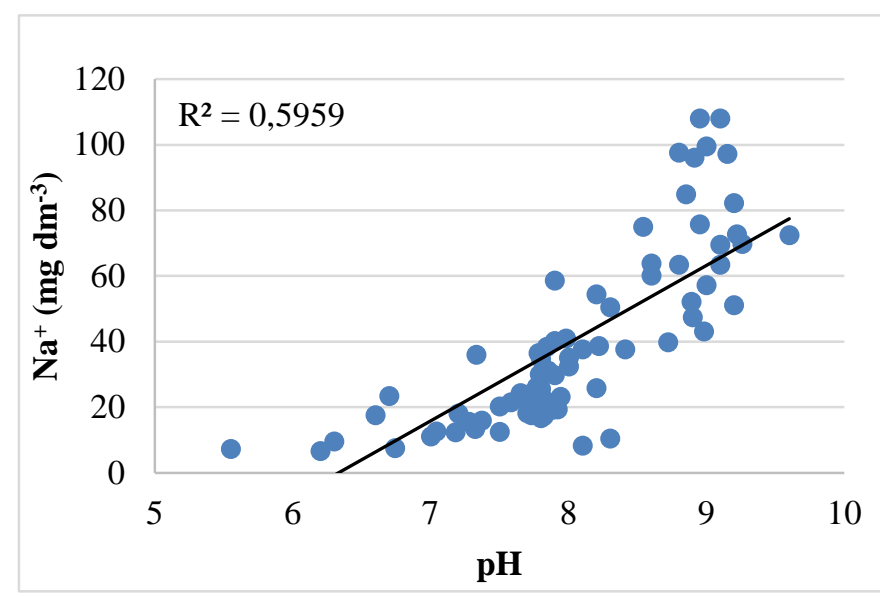

Figura 6. Correlações entre teores do isótopo ${ }^{18} \mathrm{O}$ e de íon sódio (A) e de teores de sódio com pH das águas subterrâneas (B). 
Não foi observada, entretanto, correlação entre as profundidades dos poços com as idades estimadas das águas analisadas. Amostras coletadas em poços profundos, com cerca de 300 a $450 \mathrm{~m}$, apresentaram idades variáveis, de jovens a antigas: 3.500 anos A.P (Capivari, amostra CPV-2), 5.643 A.P. (Capivari, CPV-3), 10.426 A.P. (Americana, AM-1), 19.594 (Porto Feliz, PF-1), 33.463 A.P. (Mombuca, MOM-1) e 44.645 A.P. (Monte Mor, MMOR-1).

O poço amostrado em Monte Mor (MMOR-1), que apresentou as águas mais antigas neste estudo, explota águas confinadas por camadas de rochas sedimentares siltico-argilosas como pode ser observado na Figura 7. Assim, considera-se que aspectos geológicos como a geometria do aquífero e litologias predominantes devem exercer maior influência sobre a circulação das águas, uma vez que podem restringir o fluxo subterrâneo.

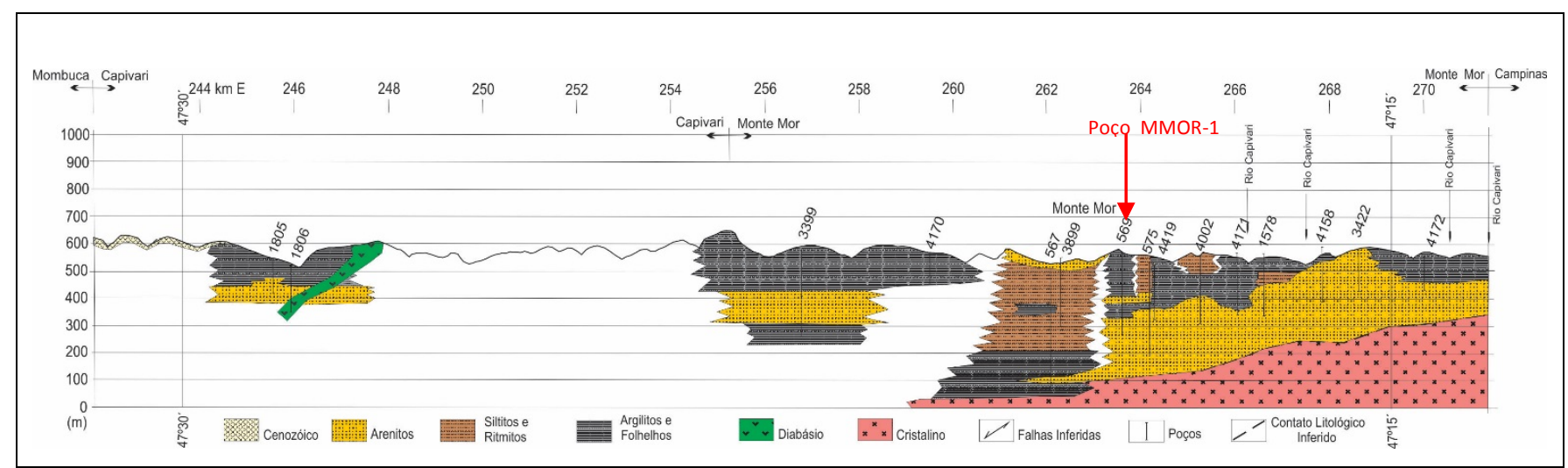

Figura 7. Seção geológica esquemática ao longo da coordenada UTMN 7.460 km, em Capivari (SP). (Extraído de: Oda et al., 2012).

No Aquífero Tubarão, a heterogeneidade litológica e descontinuidade vertical e horizontal das camadas podem constituir, desta forma, fator condicionante na idade das águas. A hipótese de evolução geoquímica de águas ao longo de fluxos subterrâneos e sua relação com o aumento das idades neste aquífero não foi confirmada e deve ser melhor avaliada.

Constatou-se, também, a necessidade de utilização de outros métodos de datação das águas subterrâneas, que permitam atingir idades mais antigas, bem como averiguar a ocorrência de misturas de águas de idades distintas.

\section{CONCLUSÃO}

Os estudos isotópicos utilizando datação por radiocarbono das águas subterrâneas do Aquífero Tubarão permitiram determinar que variam entre 1.897 e 44.763 anos A.P. na área de estudo.

A classificação hidroquímica das águas indicou três tipos principais: Ca-HCO3, Na-Ca-HCO3 e Na-HCO3, que apresentaram correlação com a composição isotópica ${ }^{2} \mathrm{H}$ e ${ }^{18} \mathrm{O}$. Os dois primeiros 
grupos estão associados a águas mais enriquecidas em isótopos estáveis, com médias de $\delta^{2} \mathrm{H}=$ $47,68 \%$ e $\delta^{18} \mathrm{O}=-7,44 \%$, enquanto as águas do terceiro grupo apresentam valores mais negativos com médias de $\delta^{2} \mathrm{H}=-58,37 \%$ e $\delta^{18} \mathrm{O}=-8,65$ \%. Estes agrupamentos podem indicar variações climáticas na época de suas recargas.

As águas do grupo 3 (Na-HCO3) estão relacionadas a poços de maior profundidade e abrangem os registros mais antigos de idades estimadas. Constatou-se, entretanto, que a maior profundidade não é fator condicionante para a ocorrência das maiores idades e que estas sofrem influência da geometria das camadas e litologias, que condicionam a circulação subterrânea.

\section{REFERÊNCIAS}

APHA/AWWA/WEF. American Public Health Association /American Water Works Association / Water Environment Federation. 1995. Standard methods for the Examination of Water and Wastewater. 19th Edition. Washington, American Public Health Association,

ARAVENA, R.; WASSENAAR, L. ; PLUMMER, L.N. 1995. Carbon 14 Groundwater ages with Methanogenesis. Water Resources Research, 31(9): 2307-2317.

DAEE-UNESP. Departamento de Águas e Energia Elétrica - Universidade Estadual Paulista 1980. Mapeamento faciológico do Subgrupo Tubarão. Convênio DAEE-UNESP, Escala 1:50.000.

DAEE - Departamento de Águas e Energia Elétrica. 1981. Estudo de Águas Subterrâneas. Região Administrativa 5. Campinas. São Paulo, DAEE, 2 v.

DAEE - Departamento de Águas e Energia Elétrica. 1982. Estudo de Águas Subterrâneas. Região Administrativa 4. Sorocaba. São Paulo, DAEE, 2 v.

DAEE-UNESP. Departamento de Águas e Energia Elétrica - Universidade Estadual Paulista. 2013. Regionalização de diretrizes de utilização e proteção das águas subterrâneas.

DAEE/ IG/ IPT/CPRM. 2005. Mapa de Águas Subterrâneas do Estado de São Paulo. Escala 1:1.000.000. Nota explicativa. São Paulo. São Paulo: DAEE-Departamento de Águas e Energia Elétrica, IG- Instituto Geológico, IPT-Instituto de Pesquisas Tecnológicas, CPRM-Serviço Geológico do Brasil, 3 v. (mapa e CD-ROM).

DINIZ, H.N. 1990. Estudo hidrogeológico do Subgrupo Itararé no médio rio Tietê, município de Tietê, SP. Dissertação de Mestrado. Instituto de Geociências, Universidade de São Paulo, São Paulo, $118 \mathrm{p}$.

EZAKI, S. 2011. Hidrogeoquímica dos Aquíferos Tubarão e Cristalino na Região de Salto (SP). São Paulo, 180 p. Tese de Doutorado. Instituto de Geociências, Universidade de São Paulo.

FERREIRA, L.M.R.; IRITANI, M.A.; ODA, G.H. 2005. Proteção de aquífero no Município de Tietê - SP. In: ABAS, Encontro Nacional de Perfuradores de Poços, 14/ Simpósio de Hidrogeologia do Sudeste, 2, Ribeirão Preto, Anais, 18 p.,CD-ROM.

HIRATA, R.; GESICKI, A.; SRACEK, O.; BERTOLO, R.; GIANNINI, P.C.; ARAVENA, R. 2011. Relation between sedimentary framework and hydrogeology in the Guarani Aquifer System in São Paulo State, Brazil. Journal of South American Earth Sciences, 31: 444-456.

IG. INSTITUTO GEOLÓGICO. 1990. Subsídios do meio físico-geológico ao planejamento da região do Município de Sorocaba (SP). São Paulo: IG/SMA. 1v. e mapas (Relatório Técnico do Instituto Geológico). 
IG. INSTITUTO GEOLÓGICO. 1991. Subsídios do meio físico-geológico ao planejamento do Município de Itu (SP). São Paulo: IG/SMA. 2 v. (Relatório Técnico do Instituto Geológico).

IG. INSTITUTO GEOLÓGICO. 1993. Subsídios do meio físico-geológico ao planejamento do Município de Campinas (SP). Programa Cartas Geológicas e Geotécnicas para o Planejamento Ambiental na Região de Sorocaba e Campinas. São Paulo: IG/SMA, 3v.

IG. INSTITUTO GEOLÓGICO. 1995. Subsídios para o planejamento regional e urbano do meio físico na porção média da bacia do Rio Piracicaba. São Paulo, Instituto Geológico - Secretaria do Meio Ambiente, Relatório Técnico.

IRITANI, M.A.; ODA, G.H.; EZAKI, S.; VARNIER, C.L. 2009. Delimitação de áreas de proteção das águas subterrâneas no Município de Capivari (SP). Revista do Instituto Geológico, 30(1/2): $1-18$.

MANIAKAS, S. 1986. Estudos geofísicos integrados à geologia da Bacia Hidrográfica do Baixo Rio Capivari-SP (Subgrupo Itararé e intrusivas associadas). Tese de doutorado. São Paulo, Instituto de Geociências, Universidade de São Paulo. 180p.

MONTANHEIRO, F.K.; CHANG, H. K.; GASTMANS, D. 2014. Estudo hidroquímico do sistema aquífero tubarão (SAT) no município de Americana, SP. Revista do Instituto Geológico, 35(1): 31-45.

ODA, G.H. 1998. Contribuição à hidrogeologia da região entre Salto de Pirapora e Itu (SP): análise da produtividade, ocorrência e circulação das águas subterrâneas dos sistemas aquíferos Tubarão e Cristalino. Dissertação de Mestrado, Instituto de Geociências, Universidade de São Paulo.São Paulo, p.

ODA, G.H.; TAKEUCHI, D.M.; IRITANI, M.A.; VARNIER, C.; ROSSINI-PPENTEADO, D.; LIMA, C.V.F.; SILVA, A.H; ZUCA, N.L.; PACHECO, R.P. 2012. Geometria do Aquífero Tubarão entre os municípios de Indaiatuba e Capivari (SP). Revista do Instituto Geológico, 33(1): 23-40.

ODA, G.H.; EZAKI, S.; IRITANI, M.A.; ROSSINI-PPENTEADO, D. 2013. Identificação de Áreas Potenciais de Restrição e Controle de Captação e Uso das Águas Subterrâneas na Porção Sul da UGRHI 05 - Projeto ARC-TUB1. São Paulo, IG/SMA, 2v. (Relatório Técnico do Instituto Geológico). Disponível em http://www.comitespcj.org.br/images/Download/ContratoFehidro450-2006_Proj-ARC-Tub1_Relatorio.pdf

PETRI, S.; PIRES, F.A. 1992. O Subgrupo Itararé (Permocarbonífero) na região do Médio Tietê, Estado de São Paulo. Revista Brasileira de Geociências, 22(3): 301-310.

SALVETTI, R. A. P. 2005. Sistemas deposicionais e paleogeografia do Subgrupo Itararé (Neopaleozóico da Bacia do Paraná), na Região entre Itu e Indaiatuba, SP. Mestrado (Dissertação). São Paulo, Instituto de Geociências - USP. 100 p.

SANTOS, P.R. 1996. Condicionamento geológico da sedimentação glacial: o Neopaleozóico da Bacia do Paraná e o Terciário da Antártida. Tese de Livre Docência. São Paulo, Instituto de Geociências - USP, 111 p.

SILVA, A.F. 2000. Potencial de água explotável, características hidráulicas e físico-químicas do aquífero Itararé na Região de Tietê - SP. Dissertação de Mestrado, IGCE-UNESP, Rio Claro, 40 p.

STEVAUX, J.C.; SOUZA FILHO, E.E.; TEIXEIRA, J.A.; LANDIM, P.M.B. 1987. Sistemas deposicionais do Subgrupo Itararé (P-CP na bacia hidrográfica do baixo Rio Capivari (SP): um modelo de prospecção de água subterrânea. In: Simpósio Regional de Geologia, 6, Rio Claro, 1987. Atas... Rio Claro, SBG, v.1, p.335-374. 
TEIXEIRA, A.L.; SALLUN, A.E.M.; SALLUN FILHO, W; DEL MONTE, M.; SILVA, R.E.N.; MATOS, J.C.V.S.R. 2009. Publicação em mídia eletrônica das cartas geológicas executadas pelo Instituto Geológico (SMA/SP) para subsidiar a ocupação e uso do meio físico na região entre

Sorocaba e Campinas. IG, São Paulo, CD-ROM. Disponível em http://www.igeologico.sp.gov.br.

VIDAL, A.C. 2002. Estudo hidrogeológico do aquífero Tubarão na área de afloramento da porção central do Estado de São Paulo. Tese de Doutorado, IGCE-UNESP, Rio Claro, 122 p.

VIDAL, A.C.; ROSTIROLLA, S.P.; CHANG, H.K. 2005. Análise de favorabilidade para a exploração de água subterrânea na Região do Médio Tietê, Estado de São Paulo. Revista Brasileira de Geociências, 35(4): 475-481.

YOSHINAGA-PEREIRA, S.Y. 1997. Proposta de representação cartográfica na avaliação para estudo de planejamento e meio ambiente. Exemplo da Região Metropolitana de Campinas-SP. São Paulo. Tese de Doutorado. Instituto de Geociências, Universidade de São Paulo. 190 p.

ZALÁN, P. V., WOLF, S., CONCEIÇÃO, J. C. J., ASTOLFI, M. A. M., VIEIRA, I. S., APPI, V. T., ZANOTTO, O. A., MARQUES, A. 1991. Tectonics and sedimentation of the Paraná Basin. Proc. Intern. Gondwana Symp., $7^{\text {th }}, 83-117$.

\section{AGRADECIMENTOS}

Ao CNPq pelo Auxílio à Pesquisa (Processo: 473194/2013-9) e pelas bolsas de iniciação científica PIBIC dos alunos do Curso de Geologia do IGc/USP César Miyata e Henrique Barbosa Jardim. Aos proprietários de poços que autorizaram e auxiliaram na coleta de amostras (DAE-Santa Bárbara D’Oeste, DAE-Americana, SAAE-Capivari, SAAE-Porto Feliz, SABESP-Monte Mor, SABESP-Elias Fausto, SABESP-Hortolândia, SABESP-Monte Mor, SABESP-Mombuca, Prefeitura Municipal de Rafard, SAMAE-Tietê, DAE-Sumaré, e particulares). Ao técnico de apoio do IG/SMA, José Alves Andrade, pelo auxílio nos trabalhos de campo e laboratoriais. 